\title{
ASYMPTOTIC STABILITY OF PLANAR RAREFACTION WAVES FOR VISCOUS CONSERVATION LAWS IN SEVERAL DIMENSIONS
}

\author{
ZHOUPING XIN
}

\begin{abstract}
This paper concerns the large time behavior toward planar rarefaction waves of the solutions for scalar viscous conservation laws in several dimensions. It is shown that a planar rarefaction wave is nonlinearly stable in the sense that it is an asymptotic attractor for the viscous conservation law. This is proved by using a stability result of rarefaction wave for scalar viscous conservation laws in one dimension and an elementary $L^{2}$-energy method.
\end{abstract}

\section{INTRODUCTION}

We will establish the asymptotic stability of planar rarefaction waves for scalar viscous conservation laws in two or more space dimensions. We consider $n$-dimensional scalar viscous conservation laws of the form

$$
u_{t}+\sum_{i=1}^{n}\left(f_{i}(u)\right)_{x_{i}}=\sum_{i, j=1}^{n} a_{i j} u_{x_{i} x_{j}}, \quad x \in \mathbf{R}^{n}, t>0,
$$

where $u \in \mathbf{R}^{1}, A=\left(a_{i j}\right)$, called the viscosity matrix, is a constant positive definite matrix, and we assume that all the flux functions are smooth (say in $C^{n}$ ) and equation (1) is genuinely nonlinear in the $x_{1}$-direction [8], i.e., for a fixed constant $a>0$,

$$
f_{1}^{\prime \prime}(u) \geq a .
$$

The initial data for equation (1) is

$$
u(x, 0)=u_{0}(x)
$$

satisfying

$$
\lim _{x_{1} \rightarrow \pm \infty}\left\|u\left(x_{1}, \cdot\right)-u_{ \pm}\right\|_{L^{\infty}\left(\mathbf{R}^{n-1}\right)}=0
$$

where $u_{ \pm}, u_{-}<u_{+}$, are two constants. A planar rarefaction wave (in $x_{1}$ direction) $u^{r}\left(x_{1}, t\right)$ is a solution of the following initial value problem for the

Received by the editors September 27, 1988.

1980 Mathematics Subject Classification (1985 Revision). Primary 35Q99, 35K35.

Key words and phrases. Nonlinear stable, viscous conservation law, planar rarefaction wave, $L^{2}$-energy method. 
corresponding inviscid equation

$$
\begin{gathered}
u_{t}+\left(f_{1}(u)\right)_{x_{1}}=0, \quad x_{1} \in \mathbf{R}^{1}, t>0, \\
u\left(x_{1}, 0\right)=u_{0}^{r}\left(x_{1}\right),
\end{gathered}
$$

where $u_{0}^{r}\left(x_{1}\right)$ satisfies

$$
\lim _{x_{1} \rightarrow \pm \infty} u_{0}^{r}\left(x_{1}\right)=u_{ \pm}, \quad \text { and } \quad \frac{d}{d x_{1}} u_{0}^{r}\left(x_{1}\right) \geq 0, \quad \text { a.e. }
$$

Since any rarefaction waves of (5) with same end states are time asymptotically equivalent (i.e., they converge to each other in $L^{\infty}$-norm as $t$ tends to infinity [6]), for definiteness, we will study a smooth rarefaction wave $u^{r}\left(x_{1}, t\right)$ of (5) with initial data $u_{0}^{r}\left(x_{1}\right)$ which satisfies

$$
\frac{d}{d x_{1}} u_{0}^{r}\left(x_{1}\right)>0, \quad \text { and } \quad\left|\frac{d^{2}}{d x_{1}^{2}} u_{0}^{r}\left(x_{1}\right)\right| \leq k_{0} \frac{d}{d x_{1}} u_{0}^{r}\left(x_{1}\right), \quad \forall x_{1} \in \mathbf{R}^{1}
$$

for some positive constant $k_{0}$. Then our main result in this paper is the following stability theorem.

Theorem 1. Suppose that $u^{r}\left(x_{1}, t\right)$ is a smooth planar rarefaction wave with initial data $u_{0}^{r}\left(x_{1}\right) \in C^{n}\left(\mathbf{R}^{1}\right)$ satisfying (8). Then there exists a constant $\delta$ such that if

$$
\left\|u_{0}(\cdot)-u_{0}^{r}(\cdot)\right\|_{H^{(n) / 2+1}\left(\mathbf{R}^{n}\right)} \leq \delta
$$

then problem (1), (3) has a global smooth solution $u(x, t)$ satisfying

$$
\lim _{t \rightarrow \infty}\left\|u(\cdot, t)-u^{r}(\cdot, t)\right\|_{L^{\infty}\left(\mathbf{R}^{n}\right)}=0 .
$$

Remark 1 . For the case that $a_{i j}=a_{i j}(u)$, it can be shown by checking our following proof that Theorem 1 still holds under the assumption that the strength of the wave, $u_{+}-u_{-}$, is small.

Remark 2. The choice of $x_{1}$-direction is no loss of generality, since we can reduce a general situation to this case by a suitable change of coordinates.

Remark 3. In the following, we will only give a proof of Theorem 1 for the case $n=2$, since the proof for $n>2$ is identical.

Remark 4. In Theorem 1, we have no restriction on the strength of the planar rarefaction wave, this is in constrast to the complementary case of the stability of viscous scalar shock fronts in several dimensions [3] which is proved for weak waves only.

The proof of Theorem 1 is based on a stability result of rarefaction waves for scalar viscous conservation laws in one dimension and an elementary $L^{2}$ energy method. The one dimension stability of expansion waves for scalar viscous conservation laws was first established by $\mathrm{Il}^{\prime}$ in and Oleinik [4] based on a maximum principle. Another approach using the semigroup argument was 
given in [1]. Our proof of multidimension stability in Theorem 1 has more in common with the proof of stability of weak rarefaction waves for systems of viscous hyperbolic conservation laws in one dimension which has recently been studied by many authors (see $[12,13,7,9,10]$ ).

The rest of the paper is organized as follows. In $\S 1$, by making use of a stability result for rarefaction wave in one space dimension, we can construct a planar solution $U\left(x_{1}, t\right)$ for equation (1) which approximates the smooth rarefaction wave $u^{r}\left(x_{1}, t\right)$; see Lemma 2. Then, we write the solution $u(x, t)$ of (1), (3) as a perturbation of $U\left(x_{1}, t\right)$ and reduce the proof of Theorem 1 to the energy estimates on the difference between $u(x, t)$ and $U\left(x_{1}, t\right)$. The basic stability estimate and higher order estimates are given in $\S 2$ and $\S 3$. Finally, we study the existence and large time behavior of the solution of (1), (3) by applying the a priori estimate derived in $\S 3$.

\section{Preliminaries}

We begin by considering the following Cauchy problem:

$$
\left\{\begin{array}{l}
U_{t}+\left(f_{1}(U)\right)_{x_{1}}=a_{11} U_{x_{1} x_{1}}, \quad x_{1} \in \mathbf{R}^{1}, t>0, \\
U\left(x_{1}, 0\right)=u_{0}^{r}\left(x_{1}\right) .
\end{array}\right.
$$

Noting that $a_{11}>0$ and (7), by the nonlinear stability of rarefaction waves for scalar conservation laws in one space dimension (see $[4,1])$, we see that there exists a unique global (in time) smooth solution $U\left(x_{1}, t\right)$ to $(11)$ which has the centered rarefaction wave of (5) determined by data $\left(u_{-}, u_{+}\right)$as a time asymptotic state in $L^{\infty}$-norm. Since all rarefaction waves of $(5)$ with the same end states are time asymptotically equivalent in $L^{\infty}$-norm, we have

$$
\lim _{t \rightarrow \infty}\left\|U(\cdot, t)-u^{r}(\cdot, t)\right\|_{L^{\infty}\left(\mathbf{R}^{1}\right)}=0 .
$$

Furthermore, if we denote $U_{x_{1}}\left(x_{1}, t\right)$ by $w\left(x_{1}, t\right)$, then $w\left(x_{1}, t\right)$ satisfies

$$
w_{t}+f_{1}^{\prime}(U) w_{x_{1}}+f_{1}^{\prime \prime}(U) w^{2}=a_{11} w_{x_{1} x_{1}} .
$$

Since $w\left(x_{1}, 0\right)=\left(u_{0}^{r}\left(x_{1}\right)\right)_{x_{1}}>0$ by $(8)$, it follows from a maximum principle that $U\left(x_{1}, t\right)$ is strictly increasing in $x_{1}$ for each fixed $t>0$, i.e.,

$$
w\left(x_{1}, t\right)=\frac{\partial}{\partial x_{1}} U\left(x_{1}, t\right)>0, \quad \forall x_{1} \in \mathbf{R}^{1}, t>0 .
$$

In other words, this says that the characteristic fields corresponding to the solution $U\left(x_{1}, t\right)$ of $(11)$ is expansive, i.e.,

$$
\frac{\partial}{\partial x_{1}}\left[f_{1}^{\prime}\left(U\left(x_{1}, t\right)\right)\right]>0, \quad \forall x_{1} \in \mathbf{R}^{1}, t>0 .
$$

We list some properties of $U\left(x_{1}, t\right)$ in the following lemma which we will use later. 
Lemma 2. The Cauchy problem (11) has a unique smooth solution $U\left(x_{1}, t\right)$ satisfying:

(i) $\lim _{t \rightarrow \infty}\left\|U(\cdot, t)-u^{r}(\cdot, t)\right\|_{L^{\infty}\left(\mathbf{R}^{1}\right)}=0$.

(ii) $\frac{\partial}{\partial x_{1}} U\left(x_{1}, t\right)>0, \quad \forall x_{1} \in \mathbf{R}^{1}, t>0$.

(iii) There exists a positive constant $K=K\left(k_{0}\right)$ such that

$$
\left|\frac{\partial^{2}}{\partial x_{1}^{2}} U\left(x_{1}, t\right)\right| \leq K \frac{\partial}{\partial x_{1}} u\left(x_{1}, t\right), \quad \forall x_{1} \in \mathbf{R}^{1}, t \geq 0 .
$$

Proof. We have already proved (i) and (ii). For (iii), we differentiate (12) with respect to $x_{1}$ and set $\phi=w_{x_{1}}$, then we get

$$
\phi_{t}+f_{1}^{\prime}(U) \phi_{x_{1}}+3 f_{1}^{\prime \prime}(U) w \phi+f_{1}^{\prime \prime \prime}(U) w^{3}=a_{11} \phi_{x_{1} x_{1}} ;
$$

thus

$$
\phi_{t}-a_{11} \phi_{x_{1} x_{1}}+f_{1}^{\prime}(U) \phi_{x_{1}}+3 f_{1}^{\prime \prime}(U) w \phi=-f_{1}^{\prime \prime \prime}(U) w^{3} .
$$

If we define a linear parabolic differential operator $L$ by

$$
L=\frac{\partial}{\partial t}-a_{11} \frac{\partial^{2}}{\partial x_{1}^{2}}+f_{1}^{\prime}(U) \frac{\partial}{\partial x_{1}}+3 f_{1}^{\prime \prime}(U) w,
$$

then

$$
L(\phi)=-f_{1}^{\prime \prime \prime}(U) w^{3} .
$$

On other hand, we get from (12) that for any constant $C$

$$
(C w)_{t}+f_{1}^{\prime}(U)(C w)_{x_{1}}+f_{1}^{\prime \prime}(U) C w^{2}=a_{11}(C w)_{x_{1} x_{1}},
$$

so that

$$
L(C w)=2 C f_{1}^{\prime \prime}(U) w^{2} .
$$

We now choose $|C|=K$ so large that

$$
K \geq \max \left\{k_{0}, \frac{1}{2 a} \max _{x_{1} \in \mathbf{R}^{\prime}}\left|f_{1}^{\prime \prime \prime}(U) w\right|\right\} .
$$

We note that we can take $K<\infty$, since it can be proved that

$$
\|w(\cdot, t)\|_{L^{\infty}\left(\mathbf{R}^{\prime}\right)} \leq M_{0}, \quad \text { for all } t \geq 0,
$$

where

$$
M_{0}=\|w(\cdot, 0)\|_{L^{\infty}\left(\mathbf{R}^{1}\right)}=\left\|\frac{d}{d x_{1}} u_{0}^{r}(\cdot)\right\|_{L^{\infty}\left(\mathbf{R}^{1}\right)},
$$

which is a finite constant by our assumption. To see this, let $M(t)$ be the solution of the following problem

$$
\frac{d}{d_{t}} M(t)+a M(t)^{2}=0, \quad M(0)=M_{0},
$$


where $a$ is the positive constant in (2). Then we have that $0 \leq M(t) \leq M_{0}$. Using (2), it is easy to check that $M(t)$ is an upper solution for nonlinear parabolic equation (12). Consequently we have

$$
\|w(\cdot, t)\|_{L^{\infty}\left(\mathbf{R}^{1}\right)} \leq M(t) \leq M_{0}, \quad \text { for all } t \geq 0 .
$$

It follows from this and (17), (18) that

$$
\begin{aligned}
L(-K w) & =-2 K f^{\prime \prime}(U) w^{2} \leq-2 K a w^{2} \leq-\left(f_{1}^{\prime \prime \prime}(U) w\right) w^{2} \\
& =L(\phi) \leq 2 K a w^{2} \leq 2 K f_{1}^{\prime \prime}(U) w^{2}=L(K w) .
\end{aligned}
$$

Since we also have $\left|\phi\left(x_{1}, 0\right)\right| \leq k_{0} w\left(x_{1}, 0\right) \leq K w\left(x_{1}, 0\right)$ by (8), thus by a comparison theorem for a parabolic equation (cf. [11]), we have

$$
\left|\phi\left(x_{1}, t\right)\right| \leq K w\left(x_{1}, t\right), \quad \forall x_{1} \in \mathbf{R}^{1}, t \geq 0 .
$$

This completes the proof of (iii). We remark that (iii) also can be proved by the argument given in [14].

By (i) in Lemma 1, we see that, in order to prove Theorem 1, it would suffice to show that the smooth expansive planar wave $U\left(x_{1}, t\right)$ is an asymptotic attractor for the equation (1). Thus we will consider the solutions of (1), (3) in a neighborhood of $U\left(x_{1}, t\right)$. As we will see later, the advantage of using $U\left(x_{1}, t\right)$ instead of $u^{r}\left(x_{1}, t\right)$ is that $U\left(x_{1}, t\right)$ is an exact solution of (1) and this will enable us quite easily to estimate some terms which do not decay in $x_{2}$-direction.

Now, we suppose that $u\left(x_{1}, x_{2}, t\right)$ is a solution of (1), (3). We decompose the solution as

$$
u\left(x_{1}, x_{2}, t\right)=U\left(x_{1}, t\right)+V\left(x_{1}, x_{2}, t\right) .
$$

It follows from (11) and (9) that the Cauchy problem (1), (3) is equivalent to the following initial value problem:

$$
\begin{gathered}
V_{t}+\left(f_{1}^{\prime}(U) V\right)_{x_{1}}+[Q(U, V)]_{x_{1}}+\left(f_{2}(U+V)\right)_{x_{2}}=\sum_{i, j=1}^{2} a_{i j} V_{x_{i} x_{j}}, \\
V\left(x_{1}, x_{2}, 0\right)=V_{0}\left(x_{1}, x_{2}\right) \equiv u_{0}\left(x_{1}, x_{2}\right)-u_{0}^{r}\left(x_{1}\right) \in H^{2}\left(\mathbf{R}^{2}\right),
\end{gathered}
$$

where $Q(U, V)=f_{1}(U+V)-f_{1}(U)-f_{1}^{\prime}(U) V$ satisfying $|Q(U, V)| \leq C V^{2}$ for some constant $C>0$ if $|V|$ is small enough. Then, we need only to show that the Cauchy problem (20), (21) has a smooth global solution, which tends to zero as $t$ approaches infinity uniformly with respect to $x_{1}$ and $x_{2}$. This is an initial value problem for a parabolic equation with initial data in $H^{2}\left(\mathbf{R}^{2}\right)$, so the local (in time) existence of solution is standard, and in order to get the global existence and large time behavior, we will need an a priori estimate on the solution of (20), (21). For this, we first define the solution space for (20) by

$$
X(0, T)=\left\{\phi\left(x_{1}, x_{2}, t\right) \in C^{0}\left(0, T ; H^{2}\right) ; \phi_{x_{1}}, \phi_{x_{2}} \in L^{2}\left(0, T ; H^{2}\right)\right\}
$$


with $T>0$. We suppose that the solution $V\left(x_{1}, x_{2}, t\right)$ of $(20),(21)$ belongs to $X(0, T)$ for some $T>0$ and set

$$
N(t) \equiv \sup _{\tau \in[0, t]}\|V(\cdot, \tau)\|_{H^{2}\left(\mathbf{R}^{2}\right)} .
$$

In what follows, we always assume that $N(T) \leq \varepsilon_{0}$ for some positive constant $\varepsilon_{0}$. The desired a priori estimate on $V$ will be derived in the following sections.

\section{BASIC ESTIMATE}

In this section we derive the basic $L^{2}$-energy estimate on $V\left(x_{1}, x_{2}\right)$. For ease of notation, in what follows we will use $\|\cdot\|$ to denote the norm in $L^{2}\left(\mathbf{R}^{2}\right)$ and $\iiint$ the triple integral over $[0, t] \times \mathbf{R}^{2}$ unless otherwise stated. Multiplying (20) by $V$ and integrating over $[0, t] \times \mathbf{R}^{2}$ gives

$$
\begin{aligned}
\frac{1}{2}\|V(\cdot, t)\|^{2} & +\iiint V\left[f_{1}^{\prime}(U) V\right]_{x_{1}} d x_{1} d x_{2} d \tau \\
& +\iiint V[Q(U, V)]_{x_{1}} d x_{1} d x_{2} d \tau \\
& +\iiint V\left[f_{2}(U+V)\right]_{x_{2}} d x_{1} d x_{2} d \tau \\
= & \frac{1}{2}\|V(\cdot, 0)\|^{2}+\iiint V \sum_{i, j=1}^{2} a_{i j} V_{x_{i} x_{j}} d x_{1} d x_{2} d \tau .
\end{aligned}
$$

Each term in (24) will be estimated separately as follows. First, by assumption that $A=\left(a_{i j}\right)$ is positive definite, we see that there exists a constant $b>0$ such that

$$
\iiint V \sum_{i, j=1}^{2} a_{i j} V_{x_{1} x_{j}} d x_{1} d x_{2} d \tau \leq-b \int_{0}^{t}\left\|\left(V_{x_{1}}, V_{x_{2}}\right)(\cdot, \tau)\right\|^{2} d \tau
$$

as follows from integrating by parts. Next, taking into account (2) and (14), we may integrate by parts twice to get

$$
\begin{aligned}
& \iiint V\left[f_{1}^{\prime}(U) V\right]_{x_{1}} d x_{1} d x_{2} d \tau \leq-\iiint f_{1}^{\prime}(U) V V_{x_{1}} d x_{1} d x_{2} d \tau \\
& \quad=\frac{1}{2} \iiint\left[f_{1}^{\prime}(U)\right]_{x_{1}} V^{2} d x_{1} d x_{2} d \tau \\
& \quad \geq \frac{a}{2} \iiint\left|U_{x_{1}}\right| V^{2}\left(x_{1}, x_{2}, t\right) d x_{1} d x_{2} d \tau .
\end{aligned}
$$

To estimate the third term on the left-hand side of (24), we write $Q(U, V)=$ $g(U, V) V^{2}$, here $g(U, V)$ is a smooth bounded function by the Taylor for- 
mula. Thus, integration by parts leads to

$$
\begin{gathered}
\iiint V[Q(U, V)]_{x_{1}} d x_{1} d x_{2} d \tau=-\iiint g(U, V) V^{2} V_{x_{1}} d x_{1} d x_{2} d \tau \\
\iiint g_{u} U_{x_{1}}\left(V^{3} / 3\right) d x_{1} d x_{2} d \tau+\iiint g_{v} V_{x_{1}}\left(V^{3} / 3\right) d x_{1} d_{x_{2}} d \tau \\
\leq C_{1} \sup \left|V\left(x_{1}, x_{2}, t\right)\right| \iiint U_{x_{1}} V^{2} d x_{1} d x_{2} d \tau \\
+C_{2} \iiint\left|V_{x_{1}} V^{3}\right| d x_{1} d x_{2} d \tau
\end{gathered}
$$

for some positive constants $C_{1}$ and $C_{2}$, where the sup is taken over $[0, t] \times$ $\mathbf{R}^{2}$. We treat the last term on the right-hand side of (27) as follows: for fixed $\left(x_{1}, x_{2}, t\right)$, we have by Cauchy inequality that

$$
\begin{aligned}
V^{2}\left(x_{1}, x_{2}, t\right) & =\int_{-\infty}^{x_{1}} \frac{\partial}{\partial x_{1}} V^{2}\left(x_{1}, x_{2}, t\right) d x_{1} \leq 2 \int_{-\infty}^{+\infty}\left|V V_{x_{1}}\right| d x_{1} \\
& \leq 2\left\|V\left(\cdot, x_{2}, \tau\right)\right\|_{L^{2}\left(\mathbf{R}^{1}\right)}\left\|V_{x_{1}}\left(\cdot, x_{2}, \tau\right)\right\|_{L^{2}\left(\mathbf{R}^{1}\right)}
\end{aligned}
$$

consequently

$$
\begin{aligned}
& \iiint\left|V_{x_{1}} V^{3}\right| d x_{1} d x_{2} d \tau=\iiint\left|V_{x_{1}} V\right| V^{2} d x_{1} d x_{2} d \tau \\
& \leq 2 \iint\left[\left\|V\left(\cdot, x_{2}, \tau\right)\right\|_{L^{2}\left(\mathbf{R}^{1}\right)}\left\|V_{x_{1}}\left(\cdot, x_{2}, \tau\right)\right\|_{L^{2}\left(\mathbf{R}^{1}\right)} \int\left|V V_{x_{1}}\right| d x_{1}\right] d x_{2} d \tau \\
& \leq 2 \iint\left\|V\left(\cdot, x_{2}, \tau\right)\right\|_{L^{2}\left(\mathbf{R}^{1}\right)}^{2}\left\|V_{x_{1}}\left(\cdot, x_{2}, \tau\right)\right\|_{L^{2}\left(\mathbf{R}^{1}\right)}^{2} d x_{2} d \tau \\
& \quad \leq 2 \sup _{0 \leq \tau \leq t, x_{2} \in \mathbf{R}^{1}}\left\|V\left(\cdot, x_{2}, \tau\right)\right\|_{L^{2}\left(\mathbf{R}^{1}\right)}^{2} \int_{0}^{t}\left\|V_{x_{1}}(\cdot, \tau)\right\|^{2} d \tau,
\end{aligned}
$$

and so

$$
\begin{aligned}
& \iiint V[Q(U, V)]_{x_{1}} d x_{1} d x_{2} d \tau \\
& \leq C_{1} \sup \left|V\left(x_{1}, x_{2}, t\right)\right| \iiint U_{x_{1}} V^{2} d x_{1} d x_{2} d \tau \\
& \quad+2 C_{2} \sup _{0 \leq \tau \leq t, x_{2} \in \mathbf{R}^{1}}\left\|V\left(\cdot, x_{2} \tau\right)\right\|_{L^{2}\left(\mathbf{R}^{1}\right)}^{2} \int_{0}^{t}\left\|V_{x_{1}}(\cdot, \tau)\right\|^{2} d \tau .
\end{aligned}
$$

Finally, we estimate the last term on the left-hand side of (24). Since $U$ does not depend on $x_{2}$, we may get after integrating by parts several times that

$$
\begin{gathered}
\iiint V\left[f_{2}(U+V)\right]_{x_{2}} d x_{1} d x_{2} d \tau=\iiint f_{2}^{\prime}(U+V) V V_{x_{2}} d x_{1} d x_{2} d \tau \\
=-\frac{1}{2} \iiint f_{2}^{\prime \prime}(U+V) V^{2} V_{x_{2}} d x_{1} d x_{2} d \tau \\
=\frac{1}{6} \iiint f_{2}^{\prime \prime \prime}(U+V) V^{3} V_{x_{2}} d x_{1} d x_{2} d \tau,
\end{gathered}
$$


consequently

$$
\left|\iiint V\left[f_{2}(U+V)\right]_{x_{2}} d x_{1} d x_{2} d \tau\right| \leq C_{3} \iiint\left|V V_{x_{2}}\right| V^{2} d x_{1} d x_{2} d \tau
$$

for some positive constant $C_{3}$. Therefore, in a similar way as for the estimate (28), we have

$$
\begin{aligned}
& \left|\iiint V\left[f_{2}(U+V)\right]_{x_{2}} d x_{1} d x_{2} d \tau\right| \\
& \quad \leq 2 \sup _{0 \leq \tau \leq t, x_{1} \in \mathbf{R}^{1}}\left\|V\left(x_{1}, \cdot, \tau\right)\right\|_{L^{2}\left(\mathbf{R}^{1}\right)}^{2} \int_{0}^{t}\left\|V_{x_{2}}(\cdot, \tau)\right\|^{2} d \tau .
\end{aligned}
$$

Collecting all the estimates $(24),(25),(26),(29)$ and (30) together, we arrive at the following lemma.

Lemma 3. There exist positive constants $\varepsilon_{i}(i=1,2)$ and $C_{4}$ depending only on the flux functions and viscosity matrix, such that if

$$
\begin{gathered}
\sup _{0 \leq \tau \leq t,\left(x_{1}, x_{2}\right) \in \mathbf{R}^{2}}\left|V\left(x_{1}, x_{2}, t\right)\right| \leq \varepsilon_{1}, \\
\sup _{0 \leq \tau \leq t}\left[\sup _{x_{1} \in \mathbf{R}^{1}}\left\|V\left(x_{1}, \cdot, \tau\right)\right\|_{L^{2}\left(\mathbf{R}^{1}\right)}^{2}+\sup _{x_{2} \in \mathbf{R}^{1}}\left\|V\left(\cdot, x_{2}, \tau\right)\right\|_{L^{2}\left(\mathbf{R}^{\prime}\right)}^{2}\right] \leq \varepsilon_{2},
\end{gathered}
$$

then, for all $t \in[0, T]$, we have

$$
\begin{aligned}
& \|V(\cdot, t)\|^{2}+\iiint\left|U_{x_{1}}\right| V^{2}\left(x_{1}, x_{2}, t\right) d x_{1} d x_{2} d \tau \\
& \quad+\int_{0}^{t}\left\|\left(V_{x_{1}}, V_{x_{2}}\right)(\cdot, \tau)\right\|^{2} d \tau \leq C_{4}\|V(\cdot, 0)\|^{2} .
\end{aligned}
$$

Next, we notice that the conditions (31) and (32) will be satisfied if $N(T)$ is suitably small. In fact, it follows from Sobolev inequality that there exists an absolute constant $C_{5}$, such that

$$
\sup _{0 \leq \tau \leq t,\left(x_{1}, x_{2}\right) \in \mathbf{R}^{2}}\left|V\left(x_{1}, x_{2}, t\right)\right| \leq C_{5} N(T) .
$$

To estimate the left-hand side of (32), we set

$$
\theta\left(x_{2}, t\right)=\left\|V\left(\cdot, x_{2}, \tau\right)\right\|_{L^{2}\left(\mathbf{R}^{1}\right)}^{2},
$$

then

$$
\begin{aligned}
\theta\left(x_{2}, t\right) & \leq \int\left|\frac{\partial}{\partial x_{2}} \theta\left(x_{2}, t\right)\right| d x_{2}=\int\left|\frac{\partial}{\partial x_{2}} \int V^{2}\left(x_{1}, x_{2}, t\right) d x_{1}\right| d x_{2} \\
& \leq 2 \iint\left|V V_{x_{2}}\right|\left(x_{1}, x_{2}, t\right) d x_{1} d x_{2} \leq\|V(\cdot, t)\|^{2}+\left\|V_{x_{2}}(\cdot, t)\right\|^{2} \\
& \leq N^{2}(T) .
\end{aligned}
$$

Thus

$$
\sup _{0 \leq \tau \leq t, x_{2} \in \mathbf{R}^{1}}\left\|V\left(\cdot, x_{2}, \tau\right)\right\|_{L^{2}\left(\mathbf{R}^{1}\right)}^{2} \leq N^{2}(T)
$$


Similarly

$$
\sup _{0 \leq \tau \leq t, x_{1} \in \mathbf{R}^{\prime}}\left\|V\left(x_{1}, \cdot, \tau\right)\right\|_{L^{2}\left(\mathbf{R}^{1}\right)}^{2} \leq N^{2}(T)
$$

As a consequence of (34)-(36) and Lemma 3, we arrive at the following basic a priori estimate

Proposition 4 (a priori estimate). There exist positive constants $\delta_{0}\left(\leq \varepsilon_{0}\right)$ and $C_{4}$ independent of $T$, such that if $N(T) \leq \delta_{0}$, then the basic energy estimate (33) holds.

\section{Higher estimates}

In this section we will establish energy estimates on higher order derivatives of $U$, which will yield a time uniform estimate on $U$ with which we can obtain the global existence of solution by a standard continuity argument. These estimates will be derived by making use of the basic estimate (33). First, we estimate the first derivative of $U$ and we have

Lemma 5. There exists a constant $C_{\sigma}>0$ such that if $N(T) \leq \delta_{0}$, then

$$
\begin{aligned}
& \|V(\cdot, t)\|_{H^{1}\left(\mathbf{R}^{2}\right)}^{2}+\iiint\left|U_{x_{1}}\right| V^{2}\left(x_{1}, x_{2}, t\right) d x_{1} d x_{2} d \tau \\
& \quad+\int_{0}^{t}\left\|\left(V_{x_{1}}, V_{x_{2}}\right)(\cdot, \tau)\right\|_{H^{1}\left(\mathbf{R}^{2}\right)}^{2} d \tau \leq C_{\sigma}\|V(\cdot, 0)\|_{H^{1}\left(\mathbf{R}^{2}\right)}^{2} .
\end{aligned}
$$

Proof. Since the estimate on $V_{x_{1}}$ is similar to but somewhat easier than that on $V_{x_{2}}$, we will only derive the estimate on $V_{x_{2}}$. For this, we differentiate (20) with respect to $x_{2}$, multiply the resulting equation by $V_{x_{2}}$ and integrate over $[0, t] \times \mathbf{R}^{2}$. We find

$$
\begin{aligned}
\frac{1}{2}\left\|V_{x_{2}}(\cdot, t)\right\|^{2}+b \int_{0}^{t}\left\|\left(V_{x_{1} x_{2}}, V_{x_{2} x_{2}}\right)(\cdot, \tau)\right\|^{2} d \tau \\
\leq \frac{1}{2}\left\|V_{x_{2}}(\cdot, 0)\right\|^{2}+\left|\iiint V_{x_{2}}\left[f_{1}^{\prime}(U) V\right]_{x_{1} x_{2}} d x_{1} d x_{2} d \tau\right| \\
+\left|\iiint V_{x_{2}}[Q(U, V)]_{x_{1} x_{2}} d x_{1} d x_{2} d \tau\right| \\
\quad+\left|\iiint V_{x_{2}}\left[f_{2}(U+V)\right]_{x_{2} x_{2}} d x_{1} d x_{2} d \tau\right|
\end{aligned}
$$

We now estimate the last three terms on the right-hand side of (38). (In what follows, we will denote by $C$ any positive constant which does not depend on 
$T$.) First, using integration by parts and Cauchy inequality, we can get

$$
\begin{aligned}
& \left|\iiint V_{x_{2}}\left[f_{1}^{\prime}(U) V\right]_{x_{1} x_{2}} d x_{1} d x_{2} d \tau\right|=\left|\iiint V_{x_{2} x_{2}}\left[f_{1}^{\prime}(U) V\right]_{x_{1}} d x_{1} d x_{2} d \tau\right| \\
& =\left|\iiint V_{x_{2} x_{2}}\left[f_{1}^{\prime \prime}(U) U_{x_{1}} V+f_{1}^{\prime}(U) V_{x_{1}}\right] d x_{1} d x_{2} d \tau\right| \\
& \leq \frac{b}{6} \int_{0}^{t}\left\|V_{x_{2} x_{2}}(\cdot, \tau)\right\|^{2} d \tau+C \iiint\left|U_{x_{1}}\right| V^{2}\left(x_{1}, x_{2}, t\right) d x_{1} d x_{2} d \tau \\
& \quad+C \int_{0}^{t}\left\|V_{x_{1}}(\cdot, \tau)\right\|^{2} d \tau .
\end{aligned}
$$

Continuing,

$$
\begin{aligned}
& \left|\iiint V_{x_{2}}[Q(U, V)]_{x_{1} x_{2}} d x_{1} d x_{2} d \tau\right|=\left|\iiint V_{x_{2} x_{2}}[(U, V)]_{x_{1}} d x_{1} d x_{2} d \tau\right| \\
& \left.\leq C \iiint\left|V_{x_{2} x_{2}}\right| \subset\left|U_{x_{1}}\right| V^{2}+V^{2}\left|V_{x_{1}}\right|+\left|V V_{x_{1}}\right|\right) d x_{1} d x_{2} d \tau \\
& \leq \frac{b}{6} \int_{0}^{t}\left\|V_{x_{2} x_{2}}(\cdot, \tau)\right\|^{2} d \tau+C \iiint\left|U_{x_{1}}\right| V^{2}\left(x_{1}, x_{2}, t\right) d x_{1} d x_{2} d \tau \\
& \quad+C \int_{0}^{t}\left\|V_{x_{1}}(\cdot, \tau)\right\|^{2} d \tau
\end{aligned}
$$

where we have used estimate (34) and the assumption $N(T) \leq \varepsilon_{0}$. In a similar way, one can obtain

$$
\begin{aligned}
& \left|\iiint V_{x_{2}}\left[f_{2}(U+V)\right]_{x_{2} x_{2}} d x_{1} d x_{2} d \tau\right| \\
& \quad=\left|\iiint V_{x_{2} x_{2}} f_{2}^{\prime}(U+V) V_{x_{2}} d x_{1} d x_{2} d \tau\right| \\
& \quad \leq \frac{b}{6} \int_{0}^{t}\left\|V_{x_{2} x_{2}}(\cdot, \tau)\right\|^{2} d \tau+C \int_{0}^{t}\left\|V_{x_{1}}(\cdot, \tau)\right\|^{2} d \tau .
\end{aligned}
$$

It follows from (38)-(41) that

$$
\begin{aligned}
& \left\|V_{x_{2}}(\cdot, t)\right\|^{2}+b \int_{0}^{t}\left\|\left(V_{x_{1} x_{2}}, V_{x_{2} x_{2}}\right)(\cdot, \tau)\right\|^{2} d \tau \\
& \begin{array}{l}
\leq\left\|V_{x_{2}}(\cdot, 0)\right\|^{2}+C\left[\iiint\left|U_{x_{1}}\right| V^{2}\left(x_{1}, x_{2}, t\right) d x_{1} d x_{2} d \tau\right. \\
\left.\quad+\int_{0}^{t}\left\|\left(V_{x_{1}}, V_{x_{2}}\right)(\cdot, \tau)\right\|^{2} d \tau\right] .
\end{array}
\end{aligned}
$$

Similar estimate holds for $V_{x_{1}}$. Now, the lemma follows from (42) and Proposition 4.

Next, we estimate the second derivatives of $V$. 
Lemma 6. If $N(T) \leq \delta_{0}$, then

$$
\begin{aligned}
& \sum_{|\alpha|=2}\left\|\partial_{x}^{\alpha} V(\cdot, t)\right\|^{2}+\int_{0}^{t} \sum_{|\alpha|=3}\left\|\partial_{x}^{\alpha} V(\cdot, \tau)\right\|^{2} d \tau \\
& \leq \sum_{|\alpha|=2}\left\|\partial_{x}^{\alpha} V(\cdot, 0)\right\|^{2}+C\left[\iiint\left|U_{x_{1}}\right| V^{2}\left(x_{1}, x_{2}, t\right) d x_{1} d x_{2} d \tau\right. \\
&\left.\quad+\int_{0}^{t}\left\|\left(V_{x_{1}}, V_{x_{2}}\right)(\cdot, \tau)\right\|_{H^{1}\left(\mathbf{R}^{2}\right)}^{2} d \tau\right] .
\end{aligned}
$$

Proof. We first estimate $V_{x_{1} x_{1}}$. Differentiating (20) twice with respect to $x_{1}$ and multiplying the resulting equation by $V_{x_{1} x_{1}}$, we find

$$
\begin{aligned}
V_{x_{1} x_{1}} & \left\{V_{x_{1} x_{1} t}+\left(f_{1}^{\prime}(U) V\right]_{x_{1} x_{1} x_{1}}+\left[Q(U, V)_{x_{1} x_{1} x_{1}}+\left[f_{2}(U+V)\right]_{x_{2} x_{1} x_{1}}\right\}\right. \\
= & \sum_{i, j=1}^{2} a_{i j} V_{x_{1} x_{1}} V_{x_{i} x_{j} x_{1} x_{1}} .
\end{aligned}
$$

Integrating the above equation over $[0, t] \times \mathbf{R}^{2}$, we find after some manipulations and integration by parts that

$$
\begin{aligned}
\frac{1}{2}\left\|V_{x_{1} x_{1}}(\cdot, t)\right\|^{2}+b \int_{0}^{t}\left\|\left(V_{x_{1} x_{1} x_{1}}, V_{x_{1} x_{1} x_{2}}\right)(\cdot, \tau)\right\|^{2} d \tau \\
\leq \frac{1}{2}\left\|V_{x_{1} x_{1}}(\cdot, 0)\right\|^{2}+\iiint V_{x_{1} x_{1} x_{1}}\left[f_{2}(U+V)\right]_{x_{2} x_{1}} d x_{1} d x_{2} d \tau \\
\quad+\iiint V_{x_{1} x_{1} x_{1}}[Q(U, V)]_{x_{1} x_{1}} d x_{1} d x_{2} d \tau \\
\quad-\iiint V_{x_{1} x_{1}}\left[f_{1}^{\prime}(U) V\right]_{x_{1} x_{1} x_{1}} d x_{1} d x_{2} d \tau .
\end{aligned}
$$

We denote the last three terms on the right-hand side of (44) by $I_{1}, I_{2}$ and $I_{3}$ respectively. $I_{1}$ can be estimated quite easily as before, indeed, it follows from Cauchy inequality that

$$
\begin{aligned}
I_{1} \equiv & \iiint V_{x_{1} x_{1} x_{1}}\left[f_{2}(U+V)\right]_{x_{2} x_{1}} d x_{1} d x_{2} d \tau \\
= & \iiint V_{x_{1} x_{1} x_{1}}\left[f_{2}^{\prime}(U+V) V_{x_{2} x_{1}}+f_{2}^{\prime \prime}(U+V) U_{x_{1}} V_{x_{2}}\right. \\
& \left.\quad+f_{2}^{\prime \prime}(U+V) V_{x_{1}} V_{x_{2}}\right] d x_{1} d x_{2} d \tau \\
\leq & \varkappa \int_{0}^{t}\left\|V_{x_{1} x_{1} x_{1}}(\cdot, \tau)\right\|^{2} \tau+C(\varkappa) \int_{0}^{t}\left(\left\|V_{x_{2}}(\cdot, \tau)\right\|^{2}+\left\|V_{x_{1} x_{2}}(\cdot, \tau)\right\|^{2}\right) d \tau \\
& +C(\varkappa) \iiint\left|V_{x_{1}}\right|^{2}\left|V_{x_{2}}\right|^{2}\left(x_{1}, x_{2}, \tau\right) d x_{1} d x_{2} d \tau,
\end{aligned}
$$

where $x$ is a small positive constant to be chosen later. By Sobolev inequality, 
we have

$$
\begin{aligned}
& \iiint\left|V_{x_{1}}\right|^{2}\left|V_{x_{2}}\right|\left(x_{1}, x_{2}, t\right) d x_{1} d x_{2} d \tau \\
& \leq \iint {\left[\left\|V_{x_{1}}\left(\cdot, x_{2}, \tau\right)\right\|_{L^{2}\left(\mathbf{R}^{1}\right)}\right.} \\
&\left.\times\left\|V_{x_{1} x_{1}}\left(\cdot, x_{2}, \tau\right)\right\|_{L^{2}\left(\mathbf{R}^{1}\right)}\left\|V_{x_{2}}\left(\cdot, x_{2}, \tau\right)\right\|_{L^{2}\left(\mathbf{R}^{1}\right)}^{2}\right] d x_{2} d \tau \\
& \leq C A_{1}\left\{\int_{0}^{t}\left\|V_{x_{1} x_{1}}(\cdot, \tau)\right\|^{2} d \tau+\iint\left\|V_{x_{2}}\left(\cdot, x_{2}, \tau\right)\right\|_{L^{2}\left(\mathbf{R}^{1}\right)}^{4} d x_{2} d \tau\right\} \\
& \leq C A_{1} \int_{0}^{t}\left\|V_{x_{1} x_{1}}(\cdot, \tau)\right\|^{2} d \tau+C A_{1} B_{1}^{2} \int_{0}^{t}\left\|V_{x_{2}}(\cdot, \tau)\right\|^{2} d \tau,
\end{aligned}
$$

where

$$
\begin{aligned}
& A_{1}=\sup _{0 \leq \tau \leq T, x_{2} \in \mathbf{R}^{1}}\left\|V_{x_{1}}\left(\cdot, x_{2}, \tau\right)\right\|_{L^{2}\left(\mathbf{R}^{1}\right)}^{2}, \\
& B_{1}=\sup _{0 \leq \tau \leq T, x_{2} \in \mathbf{R}^{1}}\left\|V_{x_{2}}\left(\cdot, x_{2}, \tau\right)\right\|_{L^{2}\left(\mathbf{R}^{1}\right)}^{2} .
\end{aligned}
$$

\section{Consequently}

$$
\begin{aligned}
I_{1} \leq & \varkappa \int_{0}^{t}\left\|V_{x_{1} x_{1} x_{1}}(\cdot, \tau)\right\|^{2} d \tau+C(\varkappa)\left(1+A_{1} B_{1}^{2}\right) \int_{0}^{t}\left\|V_{x_{2}}(\cdot, \tau)\right\|^{2} d \tau \\
& +C(\varkappa) \int_{0}^{t}\left\|V_{x_{1} x_{2}}(\cdot, \tau)\right\|^{2} d \tau+C\left((\varkappa) A_{1} \int_{0}^{t}\left\|V_{x_{1} x_{1}}(\cdot, \tau)\right\|^{2} d \tau .\right.
\end{aligned}
$$

Next, we estimate $I_{2}$. Straightforward calculation gives

$$
\begin{aligned}
I_{2} & \equiv \iiint V_{x_{1} x_{1} x_{1}}[Q(U, V)]_{x_{1} x_{1}} d x_{1} d x_{2} d \tau \\
\leq & C \iiint\left|V_{x_{1} x_{1} x_{1}}\right|\left[\left|U_{x_{1}}\right|^{2} V^{2}+\left|U_{x_{1}} V_{x_{1}}\right| V^{2}+\left|U_{x_{1}} V_{x_{1}} V\right|\right. \\
& \left.\quad+\left|V_{x_{1} x_{1}}\right| V^{2}+\left|V_{x_{1} x_{1}} V\right|+V_{x_{1}}^{2} V^{2}+|V| V_{x_{1}}^{2}+V_{x_{1}}^{2}\right] d x_{1} d x_{2} d \tau \\
& +\iiint g(U, V) V_{x_{1} x_{1} x_{1}} U_{x_{1} x_{1}} V^{2} d x_{1} d x_{2} d \tau,
\end{aligned}
$$

where $g(U, V)$ is a smooth function. By Cauchy inequality, (34) and the assumption $N(T) \leq \varepsilon_{0}$, one can verify that

$$
\begin{aligned}
I_{2} \leq \varkappa & \int_{0}^{t}\left\|V_{x_{1} x_{1} x_{1}}(\cdot, \tau)\right\|^{2} d \tau \\
& +C(x)\left\{\iiint\left|U_{x_{1}}\right| V^{2}\left(x_{1}, x_{2}, t\right) d x_{1} d x_{2} d \tau+\int_{0}^{t}\left\|V_{x_{1}}(\cdot, \tau)\right\|_{H^{1}\left(\mathbf{R}^{2}\right)}^{2} d \tau\right. \\
& \left.\quad+\iiint\left|V_{x_{1}}\right|^{4}\left(x_{1}, x_{2}, \tau\right) d x_{1} d x_{2} d \tau\right\} \\
& +\iiint g(U, V) V_{x_{1} x_{1} x_{1}} U_{x_{1} x_{1}} V^{2} d x_{1} d x_{2} d \tau
\end{aligned}
$$


where $\varkappa$ is as before. We now follow the similar argument as in (45) to show

$$
\begin{aligned}
& \iiint\left|V_{x_{1}}\right|^{4}\left(x_{1}, x_{2}, \tau\right) d x_{1} d x_{2} d \tau \\
& \quad \leq C A_{1} \int_{0}^{t}\left\|V_{x_{1} x_{1}}(\cdot, \tau)\right\|^{2} d \tau+C A_{1}^{3} \int_{0}^{t}\left\|V_{x_{2}}(\cdot, \tau)\right\|^{2} d \tau .
\end{aligned}
$$

By using (iii) of Lemma 2 and (34), we can estimate the last integral on the right-hand side of (47) as follows:

$$
\begin{aligned}
& \iiint g(U, V) V_{x_{1} x_{1} x_{1}} U_{x_{1} x_{1}} V^{2} d x_{1} d x_{2} d \tau \\
& \leq \iiint\left|g(U, V) \| V_{x_{1} x_{1} x_{1}}\right|\left(k U_{x_{1}}\right) V^{2} d x_{1} d x_{2} d \tau \\
& \leq x \int_{0}^{t}\left\|V_{x_{1} x_{1} x_{1}}(\cdot, \tau)\right\|^{2} d \tau \\
& \quad+C(\varkappa) \iiint\left|U_{x_{1}}\right| V^{2}\left(x_{1}, x_{2}, t\right) d x_{1} d x_{2} d \tau
\end{aligned}
$$

so that

$$
\begin{aligned}
I_{2} \leq & 2 \varkappa \int_{0}^{t}\left\|V_{x_{1} x_{1} x_{1}}(\cdot, \tau)\right\|^{2} d \tau \\
& +C(x)\left\{\iiint\left|U_{x_{1}}\right| V^{2}\left(x_{1}, x_{2}, t\right) d x_{1} d x_{2} d \tau\right. \\
& +\left(1+A_{1}^{3}\right) \int_{0}^{t}\left\|V_{x_{2}}(\cdot, \tau)\right\|_{H^{1}\left(\mathbf{R}^{2}\right)}^{2} d \tau \\
& \left.+\left(1+A_{1}\right) \int_{0}^{t}\left\|V_{x_{1} x_{1}}(\cdot, \tau)\right\|^{2} d \tau\right\} .
\end{aligned}
$$

Finally we estimate $I_{3}$.

$$
\begin{aligned}
I_{3} \equiv & -\iiint V_{x_{1} x_{1}}\left[f_{1}^{\prime}(U) V\right]_{x_{1} x_{1} x_{1}} d x_{1} d x_{2} d \tau \\
= & \iiint V_{x_{1} x_{1} x_{1}}\left[f_{1}^{\prime}(U) V\right]_{x_{1} x_{1}} d x_{1} d x_{2} d \tau \\
\leq & C \iiint\left|V_{x_{1} x_{1} x_{1}}\right|\left[U_{x_{1}}^{2}|V|+\left|U_{x_{1}} V_{x_{1}}\right|+\left|V_{x_{1} x_{1}}\right|+\left|U_{x_{1} x_{1}} V\right|\right] d x_{1} d x_{2} d \tau \\
\leq & 2 \varkappa \int_{0}^{t}\left\|V_{x_{1} x_{1} x_{1}}(\cdot, \tau)\right\|^{2} d \tau \\
& +C(x)\left\{\iiint\left|U_{x_{1}}\right| V^{2}\left(x_{1}, x_{2}, t\right) d x_{1} d x_{2} d \tau\right. \\
& \left.\quad+\int_{0}^{t}\left\|V_{x_{1}}(\cdot, \tau)\right\|_{H^{1}\left(\mathbf{R}^{2}\right)}^{2} d \tau+\int_{0}^{t}\left\|V_{x_{1} x_{1}}(\cdot, \tau)\right\|^{2} d \tau\right\} .
\end{aligned}
$$

where we have used Cauchy inequality and (iii) of Lemma 2 as in the estimate of $I_{2}$. 
We notice that in a similar way as in the proof of (35) and (36) one can check that

$$
\begin{aligned}
& A_{1}^{2} \leq \sup _{0 \leq \tau \leq T}\left[\left\|V_{x_{1}}(\cdot, \tau)\right\|^{2}+\left\|V_{x_{1} x_{2}}(\cdot, \tau)\right\|^{2}\right] \leq N(T)^{2}, \\
& B_{1}^{2}=\sup _{0 \leq \tau \leq T}\left[\left\|V_{x_{2}}(\cdot, \tau)\right\|^{2}+\left\|V_{x_{2} x_{2}}(\cdot, \tau)\right\|^{2}\right] \leq N(T)^{2} .
\end{aligned}
$$

Thus, if we now choose $\varkappa=b / 8$, then it follows from (44), (46) and (50)-(52) that for $N(T) \leq \delta_{0}$

$$
\begin{aligned}
& \left\|V_{x_{1} x_{1}}(\cdot, t)\right\|^{2}+b \int_{0}^{t}\left\|\left(V_{x_{1} x_{1} x_{1}}, V_{x_{1} x_{1} x_{2}}\right)(\cdot, \tau)\right\|^{2} d \tau \\
& \leq V_{x_{1} x_{1}}(\cdot, 0) \|^{2}+C\left[\iiint\left|U_{x_{1}}\right| V^{2}\left(x_{1}, x_{2}, t\right) d x_{1} d x_{2} d \tau .\right. \\
& \left.\quad+\int_{0}^{t}\left\|\left(V_{x_{1}}, V_{x_{2}}\right)(\cdot, \tau)\right\|_{H^{1}\left(\mathbf{R}^{2}\right)}^{2} d \tau\right] .
\end{aligned}
$$

Similar estimates hold for $V_{x_{1} x_{2}}$ and $V_{x_{2} x_{2}}$. Thus the proof of Lemma 6 is considered complete.

Combining Lemma 6 with Proposition 5, we have proved the following timeuniform estimate

Proposition 7 (time-uniform estimate). There exists constant $C_{4}>0$ independent of $T$, such that if $N(T) \leq \delta_{0}$, then for $t \in[0, T]$, it holds that

$$
\begin{aligned}
& \|V(\cdot, t)\|_{H^{2}\left(\mathbf{R}^{2}\right)}^{2}+\iiint\left|U_{x_{1}}\right| V^{2}\left(x_{1}, x_{2}, t\right) d x_{1} d x_{2} d \tau \\
& \quad+\int_{0}^{t}\left\|\left(V_{x_{1}} V_{x_{2}}\right)(\cdot, \tau)\right\|_{H^{2}\left(\mathbf{R}^{2}\right)}^{2} d \tau \leq C_{4}\|V(\cdot, 0)\|_{H^{2}\left(\mathbf{R}^{2}\right)}^{2} .
\end{aligned}
$$

\section{ASYMPTOTIC BEHAVIOR}

The global existence of unique solutions for problem (20), (21) and its large time behavior is an immediate consequence of Proposition 6. Indeed, combining the standard theory of the existence and uniqueness of the local (in time) solution for parabolic equations with the time-uniform estimate (53), one can extend the local solution for (20), (21) globally by the usual continuity process and show that the estimate (53) holds forever (cf. $[5,3,2])$. Thus we have

Proposition 8. There exist positive constants $\delta\left(\leq \delta_{0}\right)$ and $C_{4}$ such that if $\|V(\cdot, 0)\|_{H^{2}\left(\mathbf{R}^{2}\right)}^{2}=\left\|u_{0}(\cdot)-u_{0}^{r}(\cdot)\right\|_{H^{2}\left(\mathbf{R}^{2}\right)}^{2} \leq \delta$, then the initial value problem $(20)$, (21) has a unique global solution $V \in X(0,+\infty)$ satisfying

$$
\begin{aligned}
\sup _{t \geq 0} & \|V(\cdot, t)\|_{H^{2}\left(\mathbf{R}^{2}\right)}^{2}+\int_{0}^{+\infty}\left\|U_{x_{1}}^{1 / 2} V(\cdot, t)\right\|^{2} d t \\
& +\int_{0}^{+\infty}\left\|\left(V_{x_{1}} V_{x_{2}}\right)(\cdot, t)\right\|_{H^{2}\left(\mathbf{R}^{2}\right)}^{2} d \tau \leq C_{4}\|V(\cdot, 0)\|_{H^{2}\left(\mathbf{R}^{2}\right)}^{2} \cdot
\end{aligned}
$$


To complete the proof of Theorem 1, by Proposition 8 and (i) of Lemma 2, it suffices to show that

$$
\lim _{t \rightarrow+\infty}\|V(\cdot, t)\|_{L^{\infty}\left(\mathbf{R}^{2}\right)}=0 .
$$

In order to prove (55), first, we have from the inequality (54) and the equation (20) that

$$
\int_{0}^{+\infty}\left\{\left\|\left(V_{x_{1}}, V_{x_{2}}\right)(\cdot, t)\right\|^{2}+\left|\frac{\partial}{\partial t}\left\|\left(V_{x_{1}}, V_{x_{2}}\right)(\cdot, t)\right\|^{2}\right|\right\} d t<+\infty,
$$

from which it follows

$$
\lim _{t \rightarrow+\infty}\left\|\left(V_{x_{1}}, V_{x_{2}}\right)(\cdot, t)\right\|^{2}=0 .
$$

On the other hand, we can get

$$
\left\|V(\cdot, t)_{L^{\infty}\left(\mathbf{R}^{2}\right)}^{2} \leq 2\right\| V(\cdot, t)\|\| V_{x_{2}}(\cdot, t)\|+2\| V_{x_{1} x_{2}}(\cdot, t)\|\| V_{x_{1}}(\cdot, t) \| .
$$

In fact, for any fixed $\left(x_{1}, x_{2}, t\right) \in \mathbf{R}^{2} \times \mathbf{R}^{+}$, we have

$$
\begin{aligned}
V^{2}\left(x_{1}, x_{2}, t\right) & =2 \int_{-\infty}^{x_{1}} \frac{\partial}{\partial x_{1}} V^{2}\left(x_{1}, x_{2}, t\right) d x_{1} \leq 2 \int_{-\infty}^{+\infty}|V| V_{x_{1}} \mid\left(x_{1}, x_{2}, t\right) d x_{1} \\
& \leq\left\|V\left(\cdot, x_{2}, t\right)\right\|_{L^{2}\left(\mathbf{R}^{1}\right)}^{2}+\left\|V_{x_{1}}\left(\cdot, x_{2}, t\right)\right\|_{L^{2}\left(\mathbf{R}^{1}\right)}^{2},
\end{aligned}
$$

thus

$$
\|V(\cdot, t)\|_{L^{2}\left(\mathbf{R}^{2}\right)}^{\infty} \leq \sup _{x_{2} \in \mathbf{R}^{1}}\left\|V\left(\cdot, x_{2}, t\right)\right\|_{L^{2}\left(\mathbf{R}^{1}\right)}^{2}+\sup _{x_{2} \in \mathbf{R}^{1}}\left\|V_{x_{1}}\left(\cdot, x_{2}, t\right)\right\|_{L^{2}\left(\mathbf{R}^{1}\right)}^{2} .
$$

Furthermore we have by using Cauchy inequality that

$$
\begin{aligned}
\sup _{x_{2} \in \mathbf{R}^{1}} \| V(\cdot, & \left.x_{2}, t\right)\left\|_{L^{2}\left(\mathbf{R}^{\prime}\right)}^{2}=\sup _{x_{2} \in \mathbf{R}^{1}} \int_{-\infty}^{x_{2}} \frac{\partial}{\partial x}\right\| V\left(\cdot, x_{2}, t\right) \|_{L^{2}\left(\mathbf{R}^{\prime}\right)}^{2} d x_{2} \\
& \leq 2 \iint_{\mathbf{R}^{2}}\left|V \| V_{x_{2}}\right|\left(x_{1}, x_{2}, t\right) d x_{1}, d x_{2} \\
& \leq 2\|V(\cdot, t)\|\left\|V_{x_{2}}(\cdot, t)\right\| .
\end{aligned}
$$

Similarly, one may get

$$
\sup _{x_{2} \in \mathbf{R}^{1}}\left\|V_{x_{1}}\left(\cdot, x_{2}, t\right)\right\|_{L^{2}\left(\mathbf{R}^{1}\right)}^{2} \leq 2\left\|V_{x_{1} x_{2}}(\cdot, t)\right\|\left\|V_{x_{1}}(\cdot, t)\right\|,
$$

and (57) is proved. It follows from (56) and (57) that (55) holds, therefore we have the desired asymptotic behavior (10). This completes the proof of Theorem 1.

Acknowledgments. The author would like to thank the referee for some helpful suggestions. 


\section{BIBLIOGRAPHY}

1. E. Harabetian, Rarefaction and large time behavior for parabolic equations and monotone schemes, Comm. Math. Phys. 114 (1988), 527-536.

2. J. Goodman, Nonlinear asymptotic stability of viscous shock profile for conservation laws, Arch. Rational Mech. Anal. 95 (1986), 325-344.

3. 311 (1989), 683-695.

4. A. M. II' in and O. A. Oleinik, Behavior of the solution of the Cauchy problem for certain quasilinear equations for unbounded increase of the time, Amer. Math. Soc. Transl. (2) 42 (1964), 19-23.

5. S. Kawashima and A. Mastumura, Asymptotic stability of traveling wave solutions of system for one-dimensional gas motion, Comm. Math. Phys. 101 (1985), 97-127.

6. T. P. Liu, Linear and nonlinear large time behavior of general systems of hyperbolic conservation laws, Comm. Pure Appl. Math. 30 (1977), 767-796.

7. T. P. Liu and Z. P. Xin, Nonlinear stability of rarefaction waves for compressible NavierStokes equations, Comm. Math. Phys. 118 (1988), 451-465.

8. A. Majda, Compressible fluid flow and systems of conservation laws in several space variables, Springer-Verlag, New York, 1984.

9. A. Mastumura, Asymptotics toward rarefaction wave of solutions of the Broadwell model of a discrete velocity gas, Japan J. Appl. Math. 4 (1987), 489-502.

10. A. Mastumura and K. Nishihara, Asymptotics toward the rarefaction waves of the solutions of a one-dimensional model system for compressible viscous gas, Japan J. Appl. Math. 3 (1986), 1-13.

11. J. Smoller, Shock waves and reaction diffusion equations, Springer-Verlag, New York and Berlin, 1983.

12. Z. P. Xin, Asymptotic stability of rarefaction waves for $2 \times 2$ viscous hyperbolic conservation laws, J. Differential Equations 73 (1988), 45-77.

13. Asymptotic stability of rarefaction waves for $2 \times 2$ viscous hyperbolic conservation laws-the two modes case, J. Differential Equations 78 (1989), 191-219.

14. O. A. Ladyzenskaya, V. A. Solonnikov and N. N. Ural'ceva, Linear and quasi-linear equations of parabolic type, Amer. Math. Soc., Providence, R. I., 1968.

Courant Institute of Mathematical Sciences, 251 Mercer Street, New York, New YORK 10012 